\title{
Robust topology optimization for structures under interval uncertainty
}

By

Jinglai Wu ${ }^{1,2}$, Jie Gao ${ }^{2}$, Zhen Luo ${ }^{1, *}$, Terry Brown ${ }^{1}$

${ }^{1}$ School of Electrical, Mechanical and Mechatronic Systems

University of Technology, Sydney, NSW 2007, Australia

${ }^{2}$ School of Mechanical Science \& Engineering

Huazhong University of Science \& Technology, Wuhan, Hubei 430074, China

Original submission, 2 February 2016, $\underline{\text { ADES-D-16-00103 }}$

Revised submission, 30 March 2016, ADES-D-16-00103R1

*Correspondence author

(Dr. Z. Luo, Email: zhen.luo@uts.edu.au, Tel: +61-2-9514-2994; Fax +61-2-9514-2655)

This paper is submitted for possible publication in Advances in Engineering Software. It has not been previously published, is not currently submitted for review to any other journals, and will not be submitted elsewhere during the peer review. 


\begin{abstract}
This paper proposes a new non-probabilistic robust topology optimization approach for structures under interval uncertainty, as a complementarity of the probabilistic robust topology optimization methods. Firstly, to avoid the nested double-loop optimization procedure that is time consuming in computations, the interval arithmetic is introduced to estimate the bounds of the interval objective function and formulate the design problem under the worst scenario. Secondly, a type of non-intrusive method using the Chebyshev interval inclusion function is established to implement the interval arithmetic. Finally, a new sensitivity analysis method is developed to evaluate the design sensitivities for objective functions like structural mean compliance with respect to interval uncertainty. It can overcome the difficulty due to non-differentiability of intervals and enable the direct application of gradient-based optimization algorithms, e.g. the Method of Moving Asymptotes (MMA), to the interval uncertain topology optimization problems. Several numerical examples are used to demonstrate the effectiveness of the proposed RTO method.
\end{abstract}

KEYWORDS: Robust topology optimization; Interval uncertainty; Chebyshev inclusion function 


\section{INTRODUCTION}

In the field of structural optimization, topology optimization has experienced considerable development

over the past two decades with a range of applications [1]. Topology optimization is essentially a numerical process to optimize a prescribed objective function under specific constraints by iteratively distributing a given amount of material, until the best layout of the material is obtained in the design domain. Several typical methods have been developed for topology optimization of structures, such as the homogenization method [2], the SIMP based methods [3, 4], and the level set-based methods (LSMs), e.g. [5-7], as well as the heuristic methods like the evolutionary structural optimization (ESO) method and its variants $[8,9]$.

However, the majority of current studies about the topology optimization of structures are based on the deterministic assumption, which may result in a design that cannot satisfy the expected design goal and even a design that is unfeasible, as most problems in engineering inevitably involve various uncertainties, including the manufacturing tolerance, load variations, inhomogeneity of material properties, and so on [10]. For a structure, the topological design may be quite different when uncertain factors are considered. As a result, the performance of a structure, such as robustness and reliability, is unavoidably subject to variations in practice due to various uncertainties [11, 12]. Hence, it is necessary to incorporate uncertainties into structural topology optimization problems quantitatively, in order to enhance structural safety and avoid failure in extreme working conditions.

The reliability-based design optimization (RBDO) [11, 13-15] and robust design optimization (RDO) [1619] are two main methods, which have been used to account for different uncertainties in engineering optimization. RBDO focuses on a risk-based solution taking into account the feasibility of target at expected probabilistic levels, in which the risk is commonly measured by the probabilities of failure. Thus, RBDO seeks a design that achieves a targeted probability of failure (i.e., less than some acceptable and invariably small value) and therefore ensures that the conditions that may lead to catastrophe are unlikely. The RBDO has been combined with topology optimization to deliver the so-called reliabilitybased topology optimization (RBTO) methods. For instance, Kharmanda and et al. [20] studied topology optimization of continuum structures considering uncertainties by using the first-order reliability method. In [21], a non-deterministic topology optimization methodology is proposed by using a hybrid cellular automate method combined with a decoupled RBDO approach. Luo et al. [22] proposed a RBTO method based on a multi-ellipsoid convex model for problems consisting of non-probability uncertainties [23, 24], and so on [25]. 
The RDO aims to reduce the sensitivity of the objective function with respect to uncertain parameters, so it can minimize both the mean and variation of the objective function. The application of RDO to

structural topology optimization refers to the robust topology optimization, termed as RTO which is the major focus of this research. There have been some studies investigating RTO under uncertainties of load conditions, material properties, and geometry [26]. For instance, Sigmund [27] presented a topology optimization method to include uncertainties during the fabrication of micro and nanostructures [28]. Guest et al. [29] studied a perturbation-based topology optimization method for solving problems with small uncertainty level of externally applied loads. The perturbation method [30] was also used to solve RTO problems with small uncertainty of geometry. Asadpoure and et al. [10] combined deterministic topology optimization techniques with a perturbation method for quantification of uncertainties associated with structural stiffness. The main concept of the perturbation method is to transform the original topology optimization problem under uncertainty into an augmented deterministic problem. However, the perturbation method may produce errors which cannot be ignored when the uncertainty level of parameters is relatively high.

For the continuous problems with uncertainty, the stochastic spectrum-based method is usually used to discretise the random field. Tootkaboni et al. [31] combined the polynomial chaos expansion with topology optimization, to design continuum structures to achieve robustness in presence of random uncertainties. Zhao et al. [32] considered loading uncertainty of random field by using the KarhunenLoeve expansion to characterize the random field as a reduced set of random variables. The KarhunenLoeve expansion was also used to develop robust topology optimization method [33] with random field uncertainty, in which the univariate dimension-reduction method was combined with the Gauss type quadrature sampling to calculate statistical moments of the objective function. Jansen et al. [34] discretised the random field by using the expansion optimal linear estimation method, which particularly suits for discretising random fields with a relatively large correlation length. Zhao et al. [35] proposed an efficient approach by completely separating the Monte Carlo sampling with topology optimization to solve the RTO problem of structures under loading uncertainty, which obtained the accurate calculation of the objective function.

Most of the aforementioned RTO methods are based on the theory of random field or random variables, using a combination of the first and second order statistical moments (mean and variance) of the design response as the objective function of the RTO problems. However, in engineering, how to accurately describe probability distribution functions is a challenging task, especially for variables with limited uncertainty information. In some cases, for the uncertain variables the lower and upper bounds can be more easily obtained than the evaluation of accurate probability distributions [36]. Hence, the uncertain- 
but-bounded parameters may be more suitable for describing uncertainties under some situations. When non-probabilistic parameters are used to describe the uncertain parameters, the performance under the

worst condition can be used to define the objective function of RTO problems. In [37], the RTO problem was formulated to minimize the maximum compliance induced by the worst case of an uncertain load set, which was characterised by a convex model. By constraining the Euclidean norm of the uncertain loads, the robust optimization problem was formulated as the minimization of maximum eigenvalue of an aggregated symmetric matrix, according to the Rayleigh-Ritz theorem for symmetric matrices. However, this method can only be used to handle the convex model rather than the interval uncertainty. Csébfalvi et al. [38] considered the direction of load as uncertain-but-bounded parameters to optimize the truss by using a nonlinear optimization solver the previously developed hybrid metaheuristic ANGEL [39], but it was not used in the optimization of continuous structure. Wang et al. [36] presented a hybrid genetic algorithm, which was integrated with a simple local search strategy as the worst-case-scenario of an antioptimization, to tackle structure topology optimization under interval uncertainty. However, the antioptimization method is usually time-consuming, especially for the RTO of continuum structures, which often involves a nested double-loop optimization process that is computationally expensive.

There have been some applications about the interval uncertainty analysis and optimization. Jiang et al. [40] proposed an optimization method for uncertain structures based on convex model and a satisfaction degree of interval. in which the interval analysis method was used to determine the bounds of constraints. This method was then applied to [41], but the neural network was employed to calculate the bounds of constraints. Gao et al. [42] studied the interval dynamic response of vehicle-bridge interaction systems in which the parameters of the bridge and vehicle were considered as interval variables and a heuristic optimization method (LHNPSO) was used to find the bounds of bridge displacement. The hybrid uncertainty analysis of probability and interval uncertainty was also studied in references [43, 44].

Compared to optimization algorithms, the interval arithmetic [45] is a more efficient method that can be applied to handle the interval uncertainty, but it often produces large overestimation [46, 47]. A series of techniques have been developed to control overestimation or wrapping effect induced by interval arithmetic, e.g. the Taylor series-based method [48, 49], Taylor model method [50, 51], and Chebyshev interval method [46, 47]. Due to its non-intrusive characteristic, the Chebyshev interval method can be implemented for complex models as a black-box model. The Chebyshev interval method has been applied to the optimization problem of vehicle dynamics for hardpoints coordinate with interval uncertainty [52] and truss structures for geometric dimensions with interval uncertainty [53], and demonstrated as an effective method to compress the overestimation and avoid the nested double-loop in the optimization. 
However, there has been no publication in applying interval arithmetic to the RTO problems of continuum structures, particularly due to the following numerical issue.

Besides the overestimation in the numerical implementation, there has been no effective method so far developed for commutating the derivatives of interval functions, due to non-differentiability of intervals. However, the first-order derivatives of the objective function with respect to the design variables are often required to enable the application of the gradient-based mathematical programming methods to the RTO problems. Therefore, the difficulty for computing the derivatives is another important issue in applying the interval arithmetic to the RTO problems. In this paper, the Chebyshev interval method [46, 47] will be introduced to the RTO problems of continuum structures with uncertain-but-bounded parameters. The interval functions of RTO problems will be calculated by the interval arithmetic, in order to improve computational efficiency by avoiding the nested double-loop optimization and numerical accuracy by compressing the overestimation due to interval wrapping effect. In particular, a new numerical scheme will be developed to compute the derivatives of interval functions, which makes it possible to implement the RTO problems by using many traditional but efficient gradient-based optimization algorithms.

\section{MATERIAL DENSITY BASED APPROACH FOR TOPOLOGY OPTIMIZATION}

A typical topology optimization problem is the one to find the best layout of material within a given design domain, to minimize a prescribed objective function while satisfying a set of constraints. The wellknown topological optimization design problem is the minimization of structural mean compliance. With a given amount of material, the goal of the optimization is to identify the best topology of the material distribution that stores the minimum amount of strain energy under a set of applied loads. Using the SIMP method [54], the optimization problem is typically defined as follows:

$$
\begin{array}{ll}
\min _{\boldsymbol{\rho}} & C(\boldsymbol{\rho})=\mathbf{U}^{\mathrm{T}} \mathbf{K} \mathbf{U}=\sum_{e=1}^{N}\left(\rho_{e}\right)^{p} \mathbf{u}_{e}^{\mathrm{T}} \mathbf{k}_{0} \mathbf{u}_{e} \\
\text { s.t. } & \mathrm{g}(\boldsymbol{\rho})=\frac{\sum_{e=1}^{N} \rho_{e} v_{e}}{\sum_{e=1}^{N} v_{e}}-V \leq 0, \\
& 0<\rho_{\min } \leq \rho_{e} \leq 1 .
\end{array}
$$

where $\boldsymbol{C}(\boldsymbol{\rho})$ is the compliance, $\boldsymbol{\rho}$ is the vector including all elemental design variables, $\mathbf{k}_{0}$ denotes the element stiffness matrix, $\rho_{e}$ is an entry in $\boldsymbol{\rho}$ corresponding to element $e, v_{e}$ is a quantity that gives the volume of the element $e$ when multiplied by the design variable $\rho_{e}$, and $V$ is the upper bound for the amount of material that are allowable for usage. $\rho_{\min }$ is a vector of minimal densities, $N$ is the number of elements used to discretise the design domain, and $p$ is the penalization power. $\mathbf{U}$ and $\mathbf{F}$ denote the global displacement vector and forcing vector, respectively, and they satisfy the following equation

$$
\mathbf{K U}=\mathbf{F}
$$


where $\mathbf{K}$ is the global stiffness matrix.

The above optimization model can usually be solved by several optimization algorithms, such as the Optimality Criteria (OC) method [55], the Method of Moving Asymptotes (MMA) [56], and so on. If the MMA is used, the sensitivity or the first-order derivatives of the objective function with respect to the design variables should be firstly derived, as follows [54]:

$$
h(\boldsymbol{\rho})=\frac{\partial C(\boldsymbol{\rho})}{\partial \rho_{e}}=-p\left(\rho_{e}\right)^{p-1} \mathbf{u}_{e}^{\mathrm{T}} \mathbf{k}_{0} \mathbf{u}_{e}
$$

In SIMP, the filtering techniques will normally be used to smooth the design sensivity, so as to ensure regularity or existence of solutions of a topology optimization problem without occurring checkboards $[54,57]$. If the sensitivity filtering scheme [54] id used, the element sensitivity is normally modified as

$$
\frac{\partial \hat{C}(\boldsymbol{\rho})}{\partial \rho_{e}}=\frac{1}{\rho_{e} \sum_{i \in N_{e}} w_{i}} \sum_{i \in N_{e}} w_{i} \rho_{i} \frac{\partial C(\boldsymbol{\rho})}{\partial \rho_{e}}
$$

where $\mathrm{Ne}$ is the set of elements whose center-center distance to element $e$ is no larger than the filter radius $R$, and $w_{i}$ is the weight factor, determined by the following equation

$$
w_{i}=R-\operatorname{dist}\left(\rho_{i}, \rho_{e}\right)
$$

Using Eq. (4) to compute the derivatives of the objective function, the optimization given in Eq. (1) can be solved by the gradient-based optimization algorithms, e.g. the MMA.

\section{ROBUST TOPOLOGY OPTIMIZATION UNDER INTERVAL UNCERTAINTY}

\subsection{RTO formulation under interval uncertainty}

The uncertain parameters in RTO are expressed by interval numbers in this study. Using IR to denote the interval real set, an interval number $[x] \in \mathbf{I R}$ is defined by its lower bound and upper bound, i.e.

$$
[x]=[\underline{x}, \bar{x}]=\{x \in \mathbf{R} \mid \underline{x} \leq x \leq \bar{x}\}
$$

where $\underline{x}$ and $\bar{x}$ denotes the lower bound and upper bound of interval $[x]$, respectively. Similar to the real number, the interval vector $[\mathbf{x}] \in \mathbf{I R}^{k}$ can be defined as

$$
[\mathbf{x}]=[\underline{\mathbf{x}}, \overline{\mathbf{x}}]=\left\{x_{i} \in \mathbf{R} \mid \underline{x}_{i} \leq x_{i} \leq \bar{x}_{i}, i=1,2, \ldots, k\right\}
$$

The addition and subtraction operation of interval arithmetic are defined by

$$
[x]+[y]=[\underline{x}+\underline{y}, \bar{x}+\bar{y}],[x]-[y]=[\underline{x}-\bar{y}, \bar{x}-\underline{y}]
$$

More detailed interval arithmetic operations can be found in [58]. Using interval arithmetic, the bounds of a continuous interval function $f([x]), f([x])=[\underline{f}, \bar{f}]$, with explicit expression can be calculated easily. 
When interval uncertain parameters (e.g. loads and material properties) involved in the original topology optimization model, the formulation in Eq. (1) will be re-written as follows:

$$
\begin{array}{ll}
\min _{\boldsymbol{\rho}} & C(\boldsymbol{\rho},[\mathbf{x}])=\sum_{e=1}^{N}\left(\rho_{e}\right)^{p} \mathbf{u}_{e}^{\mathrm{T}}([\mathbf{x}]) \mathbf{k}_{0}([\mathbf{x}]) \mathbf{u}_{e}([\mathbf{x}]) \\
\text { s.t. } & \mathrm{g}(\boldsymbol{\rho}) \leq 0, \\
& 0<\rho_{\min } \leq \rho_{e} \leq 1 .
\end{array}
$$

Here, $[\mathbf{x}] \in \mathbf{I R}^{k}$ denotes a $k^{\text {th }}$-dimensional vector of interval parameters. The interval parameters are not involved in the constraint of the volume fraction. In this case, the original structural mean compliance becomes an interval objective function with respect to the interval parameters [x], denoted by its lower bound $\underline{C}(\boldsymbol{\rho},[\mathbf{x}])$ and upper bound $\bar{C}(\boldsymbol{\rho},[\mathbf{x}])$. Considering the worst case of the optimization, the upper bound of the interval function will be used to define the new objective function for the RTO problem as

$$
\begin{array}{ll}
\min _{\boldsymbol{\rho}} & \bar{C}(\mathbf{\rho},[\mathbf{x}])=\max _{\mathbf{x} \in[\mathbf{x}]} \sum_{e=1}^{N}\left(\rho_{e}\right)^{p} \mathbf{u}_{e}^{\mathrm{T}}(\mathbf{x}) \mathbf{k}_{0}(\mathbf{x}) \mathbf{u}_{e}(\mathbf{x}) \\
\text { s.t. } & \mathrm{g}(\mathbf{\rho}) \leq 0, \\
& 0<\rho_{\min } \leq \rho_{e} \leq 1 .
\end{array}
$$

It is noted that the above objective function includes a maximum operation, which is computationally expensive if the optimization method is employed to compute the maximum compliance under the interval uncertainty. One may consider enumerating all the vertices of interval parameters to estimate the maximum compliance, so the optimization model will be transformed to

$$
\begin{array}{ll}
\min _{\boldsymbol{\rho}} & \bar{C}(\boldsymbol{\rho},[\mathbf{x}])=\max _{i=1, \ldots, K} \sum_{e=1}^{N}\left(\rho_{e}\right)^{p} \mathbf{u}_{e}^{\mathrm{T}}\left(\mathbf{x}_{i}\right) \mathbf{k}_{0}\left(\mathbf{x}_{i}\right) \mathbf{u}_{e}\left(\mathbf{x}_{i}\right) \\
\text { s.t. } & \mathrm{g}(\boldsymbol{\rho}) \leq 0, \\
& 0<\rho_{\min } \leq \rho_{e} \leq 1 .
\end{array}
$$

where $\mathbf{x}_{i}$ denotes the vertices of the interval parameters, and $K$ is the number of vertices of the interval parameters. This model becomes a minimax optimization problem [59] which can be solved by using the MMA algorithm. However, the optimization procedure is usually unstable, as demonstrated by the numerical examples given in Section 5. The interval arithmetic will be used to estimate the $\bar{C}(\boldsymbol{\rho},[\mathbf{x}])$.

\subsection{RTO using the Chebyshev interval method}

If the explicit expression of the objective function with respect to interval parameters [x] can be obtained, the interval arithmetic can be directly used to calculate the upper bound of the interval objective function. However, it is hard to obtain the explicit expression, so we have to solve the following linear system to obtain the interval displacement, and then calculate the interval function for the mean compliance.

$$
\mathbf{K}([\mathbf{x}]) \mathbf{U}=\mathbf{f}([\mathbf{x}])
$$


The Eq. (12) is difficult to solve by using interval arithmetic directly, especially for the large dimensional case in the finite element analysis. On the other hand, each node displacement occurs several times in the

expression of compliance, which may produce large overestimation if the interval arithmetic is used directly. To overcome these problems, the non-intrusive Chebyshev interval method will be employed to calculate the interval compliance.

For simplicity but without loss of generality, the interval vector is considered to be $[\mathbf{x}]=[-1,1]^{k}$. Based on the Chebyshev interval method [47], a general continuous interval function $F([\mathbf{x}])$ often without explicit expression can be approximated by the following $n$-th order truncated Chebyshev series:

$$
F([\mathbf{x}])=\sum_{j_{1}=0}^{n} \ldots \sum_{j_{k}=0}^{n} \frac{1}{2^{l}} f_{j_{1} \ldots j_{k}} \prod_{i=1}^{k} \cos \left(j_{i}\left[\theta_{i}\right]\right)
$$

where $\left[\theta_{i}\right]=\arccos \left(\left[x_{i}\right]\right)=[0, \pi], \cos \left(j_{i}\left[\theta_{i}\right]\right)$ denotes the Chebyshev polynomial, $l$ is the number of subscript $j_{i}=0(i=1, \ldots, \mathrm{k})$ of $f_{j_{1} \ldots j_{k}}$, which are the Chebyshev coefficients and can be calculated by the following Gaussian-Chebyshev numerical quadrature formula.

$$
\begin{aligned}
f_{j_{1}, \ldots, j_{k}} & =\left(\frac{2}{\pi}\right)^{k} \int_{0}^{\pi} \ldots \int_{0}^{\pi} F\left(\cos \theta_{1}, \ldots, \cos \theta_{k}\right) \cos j_{1} \theta_{1} \ldots \cos j_{k} \theta_{k} d \theta_{1} \ldots d \theta_{k} \\
& \approx\left(\frac{2}{m}\right)^{k} \sum_{q_{1}=1}^{m} \ldots \sum_{q_{k}=1}^{m} F\left(\cos \theta_{q_{1}}, \ldots, \cos \theta_{q_{k}}\right) \cos j_{1} \theta_{q_{1}} \ldots \cos j_{k} \theta_{q_{k}}
\end{aligned}
$$

where $m$ denotes the order of numerical quadrature formula, $\theta_{q}$ is the interpolation points of the numerical quadrature formula, determined by

$$
\theta_{q}=\frac{2 q-1}{m} \frac{\pi}{2}, q=1,2, \ldots, m
$$

Using Eq. (14), we do not need the explicit expression of function $F(x)$, but only the function values at some interpolation points, so $F(x)$ can be very complicated model or even black-box model. Once the Eq. (13) is obtained, the interval arithmetic can be used to calculate the bounds of $F([\mathbf{x}])$ based on the characteristic of trigonometric function, i.e.

$$
\cos (0[\theta])=1, \cos (j[\theta])=[-1,1], j=1,2, \ldots \text { for }[\theta]=[0, \pi]
$$

Using the interval arithmetic shown in Eq. (8), the bounds of an interval function can be estimated by

$$
F([\mathbf{x}])=\frac{1}{2^{k}} f_{0 \ldots 0}+\sum_{\substack{0 \leq j_{1}, \ldots, j_{k} \leq n \\ j_{1}+\ldots+j_{k}>0}} \frac{1}{2^{l}} f_{j_{1} \ldots j_{k}} \times[-1,1]=\frac{1}{2^{k}} f_{0 \ldots 0}+\left[-\sum_{\substack{0 \leq j_{1}, \ldots, j_{k} \leq n \\ j_{1}+\ldots+j_{k}>0}} \frac{1}{2^{l}}\left|f_{j_{1} \ldots j_{k}}\right|, \sum_{\substack{0 \leq j_{1}, \ldots, j_{k} \leq n \\ j_{1}+\ldots+j_{k}>0}} \frac{1}{2^{l}}\left|f_{j_{1} \ldots j_{k}}\right|\right]
$$

Let $C^{e}(\boldsymbol{\rho},[\mathbf{x}])$ denotes the interval element compliance under the interval parameters, i.e.

$$
C^{e}(\boldsymbol{\rho},[\mathbf{x}])=\left(\rho_{e}\right)^{p} \mathbf{u}_{e}^{\mathrm{T}}([\mathbf{x}]) \mathbf{k}_{0}([\mathbf{x}]) \mathbf{u}_{e}([\mathbf{x}])
$$


Replace the general interval function $F([\mathbf{x}])$ by the interval compliance $C^{e}(\boldsymbol{\rho},[\mathbf{x}])$ in Eq. (13), we have

$$
C^{e}(\boldsymbol{\rho},[\mathbf{x}])=\sum_{j_{1}=0}^{n} \ldots \sum_{j_{k}=0}^{n} \frac{1}{2^{l}} c_{j_{1} \ldots j_{k}}^{e}(\boldsymbol{\rho}) \prod_{i=1}^{k} \cos \left(j_{i}\left[\theta_{i}\right]\right)
$$

The coefficients $c_{j_{1} \ldots j_{k}}^{e}$ are generated by Eq. (14) after replacing $F$ by $C^{e}$. It should be noted that the coefficients will be changed along with the design variables $\boldsymbol{\rho}$, noted as $c_{j_{1} \ldots j_{k}}^{e}(\boldsymbol{\rho})$. Considering Eq. (9), the interval compliance of the structure can be expressed by

$$
C(\mathbf{\rho},[\mathbf{x}])=\sum_{e=1}^{N} C^{e}(\mathbf{\rho},[\mathbf{x}])=\sum_{e=1}^{N} \sum_{j_{1}=0}^{n} \ldots \sum_{j_{k}=0}^{n} \frac{1}{2^{l}} c_{j_{1} \ldots j_{k}}^{e}(\mathbf{\rho}) \prod_{i=1}^{k} \cos \left(j_{i}\left[\theta_{i}\right]\right)
$$

Based on Eq. (17), the objective function of the RTO problem can finally be expressed, as follows:

$$
\bar{C}(\boldsymbol{\rho},[\mathbf{x}])=\sum_{e=1}^{N}\left(\frac{1}{2^{k}} c_{0 \ldots 0}^{e}(\mathbf{\rho})+\sum_{\substack{0 \leq j_{1}, \ldots, j_{k} \leq n \\ j_{1} \ldots \ldots+j_{k}>0}} \frac{1}{2^{l}}\left|c_{j_{1} \ldots j_{k}}^{e}(\boldsymbol{\rho})\right|\right)
$$

Eq. (21) assumes that all the element interval compliances can reach their upper bounds simultaneously, so it usually makes $\bar{C}(\boldsymbol{\rho},[\mathbf{x}])$ larger than its exact upper bound. However, $\bar{C}(\boldsymbol{\rho},[\mathbf{x}])$ has the same trend as the exact upper bound, so it can be used as the objective function to replace the exact upper bound.

\section{Numerical implementation of RTO using interval arithmetic}

\subsection{Sensitivity analysis of the interval functions}

To use the MMA to solve the RTO, the derivatives of the objective with respect to the design variables have to be produced. The objective shown in Eq. (22) contains the operation of computing the absolute value, which is difficult to calculate the derivative, so we change Eq. (22) as the following equation

$$
\bar{C}(\boldsymbol{\rho},[\mathbf{x}])=\sum_{e=1}^{N}\left(\frac{1}{2^{k}} c_{0 \ldots 0}^{e}(\boldsymbol{\rho})+\sum_{\substack{0 \leq j_{1} \ldots, j_{k} \leq n \\ j_{1}+\ldots, j_{k}>0}} \frac{1}{2^{l}} c_{j_{1} \ldots j_{k}}^{e}(\boldsymbol{\rho}) \operatorname{sign}\left(c_{j_{1} \ldots j_{k}}^{e}(\boldsymbol{\rho})\right)\right)
$$

where the sign function is defined as

$$
\operatorname{sign}(x)= \begin{cases}-1, & x<0 \\ 0, & x=0 \\ 1, & x>0\end{cases}
$$

Differentiating Eq. (22) with respect to design variables $\rho_{e}$, we have the sensitivity

$$
\frac{\partial \bar{C}(\boldsymbol{\rho},[\mathbf{x}])}{\partial \rho_{e}}=\sum_{e=1}^{N}\left(\frac{\partial c_{0 . \ldots 0}^{e}(\boldsymbol{\rho})}{2^{k} \partial \rho_{e}}+\sum_{\substack{0 \leq j_{1}, j_{j} \leq n \\ j_{1} \ldots \ldots j_{k}>0}} \frac{\partial c_{j_{1}, j_{k}}^{e}(\mathbf{\rho})}{2^{l} \partial \rho_{e}}\left(\operatorname{sign}\left(c_{j_{1} \ldots j_{k}}^{e}(\boldsymbol{\rho})\right)+\frac{\partial \operatorname{sign}\left(c_{j_{1} \ldots j_{k}}^{e}(\boldsymbol{\rho})\right)}{\partial c_{j_{1} \ldots j_{k}}^{e}(\boldsymbol{\rho})} c_{j_{1} \ldots j_{k}}^{e}(\boldsymbol{\rho})\right)\right)
$$


It is noted the function ' $\operatorname{sign}(x)$ ' is not continuous, as it is not differentiable at the point $x=0$, as given in Eq. (23). Here the Heaviside projection method is used to smooth the sign function, as follows:

$$
s(x)=\tanh (\beta x)
$$

where the parameter $\beta$ is a positive real number. The $s(x)$ and the $\operatorname{sign}(x)$ are plot in Fig. 1, which shows that the $s(x)$ will be equal to $\operatorname{sign}(x)$ when the parameter $\beta$ tends to be infinite.

Replacing the $\operatorname{sign}(x)$ by the continuous $s(x)$ in Eq. (24), we can obtain the following equation

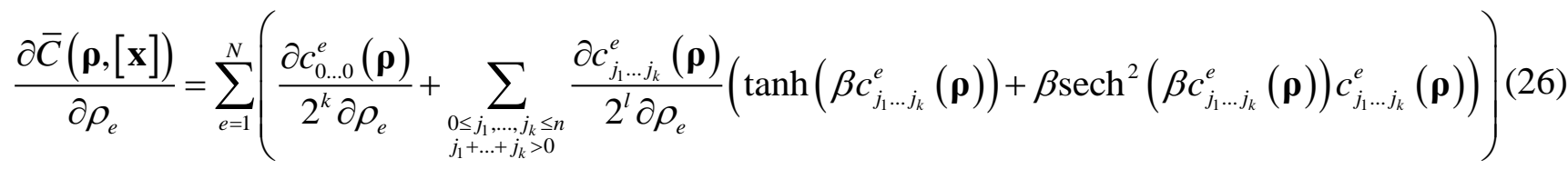

Since the first-order derivatives of the coefficients $c_{j_{1} \ldots j_{k}}^{e}$ with respect to $\rho_{e}$ (sensitivities) cannot be explicitly obtained, we will consider the following expansion, to approximate these first-order derivatives. Considering the interval parameters, the derivative of the compliance in Eq. (3) can be rewritten as

$$
h(\boldsymbol{\rho},[\mathbf{x}])=\frac{\partial C(\boldsymbol{\rho},[\mathbf{x}])}{\partial \rho_{e}}=-p\left(\rho_{e}\right)^{p-1} \mathbf{u}_{e}^{\mathrm{T}}([\mathbf{x}]) \mathbf{k}_{0}([\mathbf{x}]) \mathbf{u}_{e}([\mathbf{x}])
$$

Similar to the procedure shown in Section 3.2, the derivatives can also be expanded by using the truncated Chebyshev series, as follows:

$$
h(\boldsymbol{\rho},[\mathbf{x}])=\sum_{j_{1}=0}^{n} \ldots \sum_{j_{k}=0}^{n} \frac{1}{2^{l}} h_{j_{1} \ldots j_{k}}(\boldsymbol{\rho}) \prod_{i=1}^{k} \cos \left(j_{i}\left[\theta_{i}\right]\right)
$$

It is noted that the above equation involves the coefficients $h_{j_{1} \ldots j_{k}}(\boldsymbol{\rho})$, which actually can be directly calculated by using the same quadrature formula, as given in Eq. (14).

Differentiating Eq. (20) with respect to $\rho_{e}$, we have 


$$
\frac{\partial C(\boldsymbol{\rho},[\mathbf{x}])}{\partial \rho_{e}}=\sum_{e=1}^{N} \sum_{j_{1}=0}^{n} \ldots \sum_{j_{k}=0}^{n} \frac{\partial c_{j_{1} \ldots j_{k}}^{e}(\boldsymbol{\rho})}{2^{l} \partial \rho_{e}} \prod_{i=1}^{k} \cos \left(j_{i}\left[\theta_{i}\right]\right)
$$

Comparing Eq. (28) and Eq. (29), we will have

$$
\sum_{e=1}^{N}\left(\partial c_{j_{1} \ldots j_{k}}^{e}(\boldsymbol{\rho}) / \partial \rho_{e}\right)=h_{j_{1} \ldots j_{k}}(\boldsymbol{\rho})
$$

In this case, we can get the summation of the first-order derivatives of coefficients $c_{j_{1} \ldots j_{k}}^{e}(\boldsymbol{\rho})$ with respect to $\rho_{e}$ at the right-hand side of Eq. (26), according to Eq. (14). Hence, substituting Eq. (30) into Eq. (26), we can finally find the design sensitivity of the interval objective function as

$\frac{\partial \bar{C}(\boldsymbol{\rho},[\mathbf{x}])}{\partial \rho_{e}}=\frac{1}{2^{k}} h_{0 \ldots 0}(\boldsymbol{\rho})+\sum_{\substack{0 \leq j_{1}, \ldots j_{k} \leq n \\ j_{1} \ldots+j_{k}>0}} \frac{1}{2^{l}} h_{j_{1} \ldots j_{k}}(\boldsymbol{\rho})\left(\tanh \left(\beta c_{j_{1} \ldots j_{k}}^{e}(\boldsymbol{\rho})\right)+\beta \operatorname{sech}^{2}\left(\beta c_{j_{1} \ldots j_{k}}^{e}(\boldsymbol{\rho})\right) c_{j_{1} \ldots j_{k}}^{e}(\boldsymbol{\rho})\right)$

After obtaining Eq. (31), in a similar way as given by Eq. (4), we can still use filtering schemes, such as the sensitivity filtering method, to smooth the sensitivity before finally feeding into the MMA solver.

\subsection{Optimization procedure}

With the use of MMA algorithm the implementation process of the proposed robust topology optimization method under interval uncertainties can be summarized, as follows:

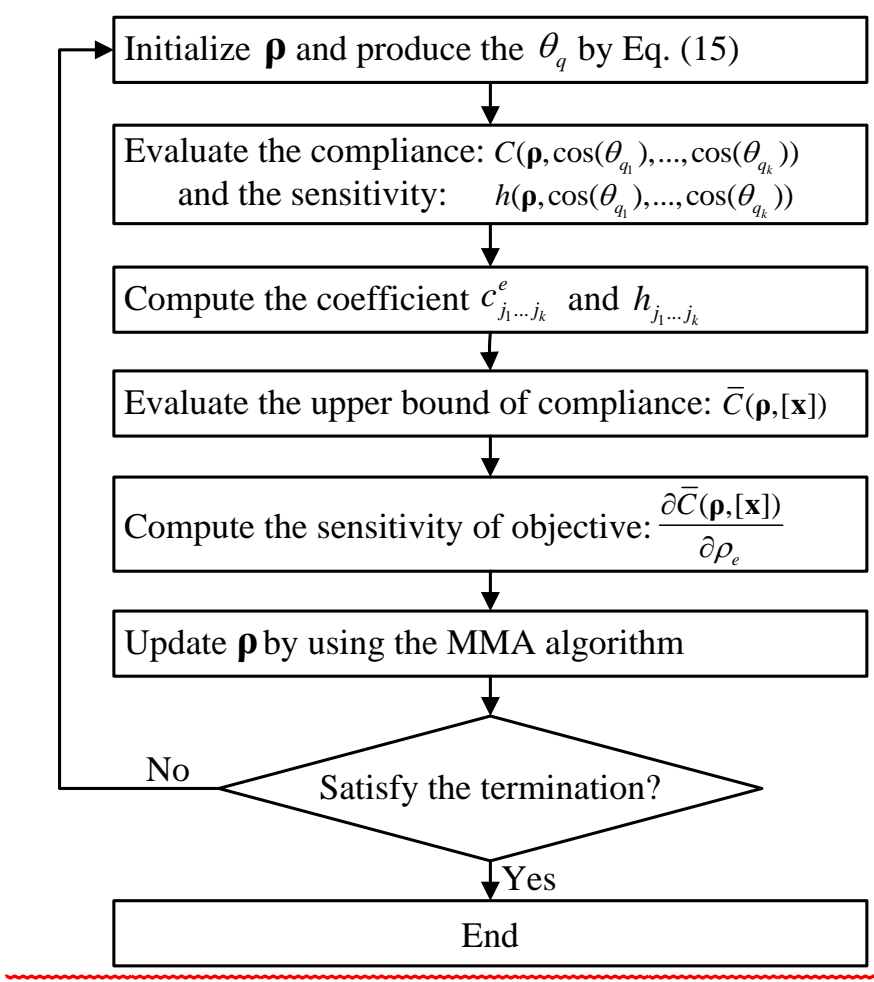

Figure. 2 The flowchart of RTO based on interval method 
This process mainly contains 6 steps that are:

1) Initialize the design variables $\boldsymbol{\rho}$ and produce the interpolation points of the interval parameters $\theta_{q}$ by

using Eq. (15);

2) Set the interval parameters to be the values at the interpolation points produced in Step (1), solve the displacements from the Eq. (12) and then evaluate the compliance $C\left(\boldsymbol{\rho}, \cos \left(\theta_{q_{1}}\right), \ldots, \cos \left(\theta_{q_{k}}\right)\right)$ and sensitivity $h\left(\boldsymbol{\rho}, \cos \left(\theta_{q_{1}}\right), \ldots, \cos \left(\theta_{q_{k}}\right)\right)$ at these interpolation points;

3) Calculate the coefficients $c_{j_{1} \ldots j_{k}}^{e}$ and $h_{j_{1} \ldots j_{k}}$ using the Gaussian quadrature formula in Eq. (14)i

4) Evaluate the upper bound of interval compliance $\bar{C}(\boldsymbol{\rho},[\mathbf{x}])$ using Eq. (21);

5) Calculate the sensitivity of the objective $\frac{\partial \bar{C}(\boldsymbol{\rho},[\mathbf{x}])}{\partial \rho_{e}}$ using Eq. (31) and then substitute it into Eq. (4) to get the final sensitivity to be used in MMA;

6) Update the design variables by the MMA, and go back to Step 2) until convergence.

\section{Numerical examples}

In this section, three numerical examples with different interval parameters will be used to illustrate the proposed RTO method under interval uncertainty. Besides the interval optimization method, the minimax optimization in Eq. (11) based on the vertices of interval parameters will also be used to implement the RTO. The traditional deterministic topology optimization is also employed to show the difference between the deterministic and uncertain topology optimization problems.

\subsection{Simple column design}

The boundary conditions and load for the design problem is shown as Fig. 3. In the optimization, the volume fraction $V$ is set as 0.3 . The magnitude of the force $F$ is set as 1 , but the direction of force is considered as an interval parameter and subject to variation. For the deterministic case, the force direction is set as $\theta=-90^{\circ}$. For the uncertain cases, three uncertainties are considered as: 1) small uncertainty $\left.[\theta]=\left[-95^{\circ},-85^{\circ}\right], 2\right)$ medium uncertainty $[\theta]=\left[-100^{\circ},-80^{\circ}\right]$, and 3) large uncertainty $[\theta]=\left[-110^{\circ},-70^{\circ}\right]$.

The mesh size is 60 by 60 , and the filter radius is set as 1.5 . The parameters of the interval optimization method are set as follows: the $2^{\text {nd }}$ order Chebyshev series $(n=2)$, the $3^{\text {rd }}$ order quadrature formula $(m=3)$, and $\beta=10$. When the maximum change of design variables of two successive iterations is smaller than 0.01 and the related change of objective function values is smaller than $0.01 \%$, or the maximum number of iteration is larger than 500, the optimization will be terminated. The minimax optimization model uses the same convergence criterion. 
It can be found that the results of the RTO problems based on the interval method are different from the

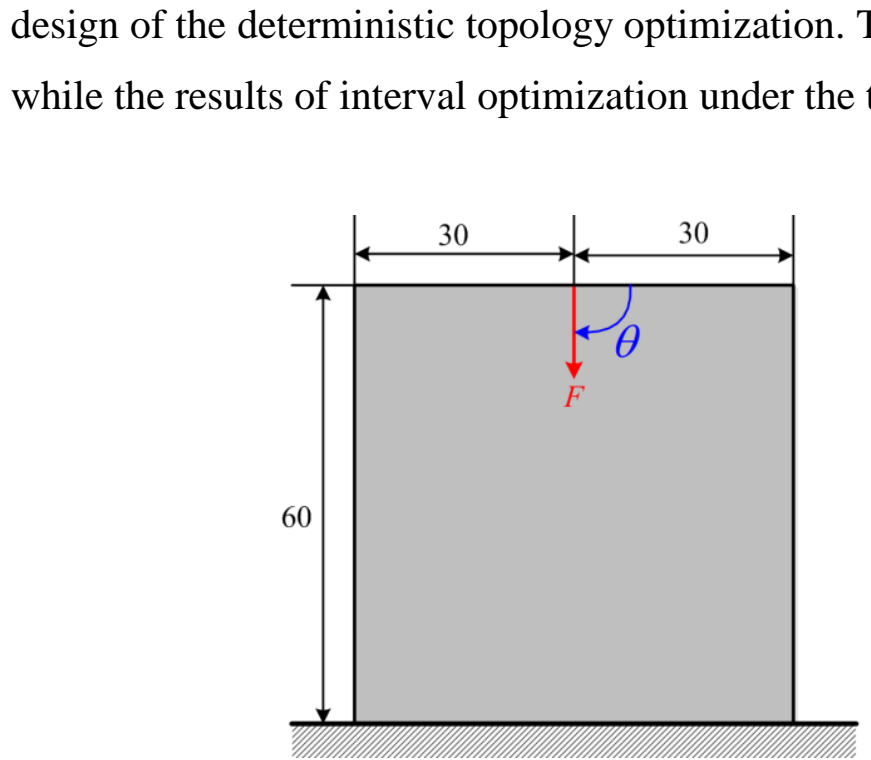

Figure. 3 Design domain

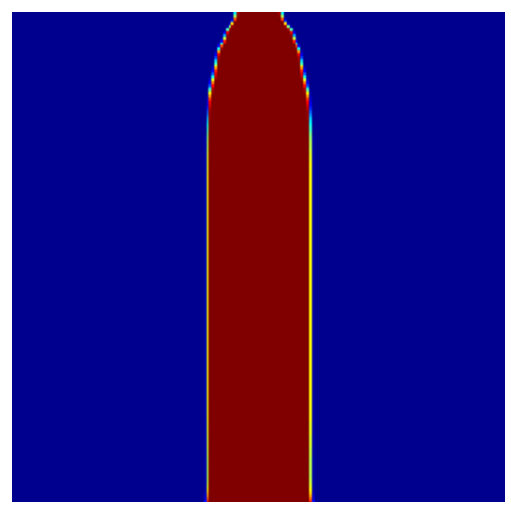

Figure. 4 Deterministic design

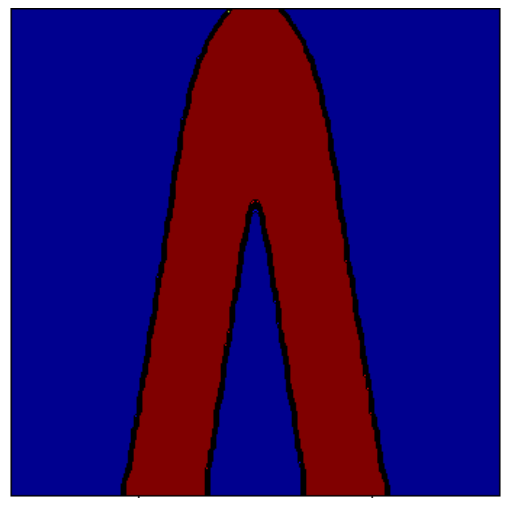

(a) $[\theta]=\left[-95^{\circ},-85^{\circ}\right]$

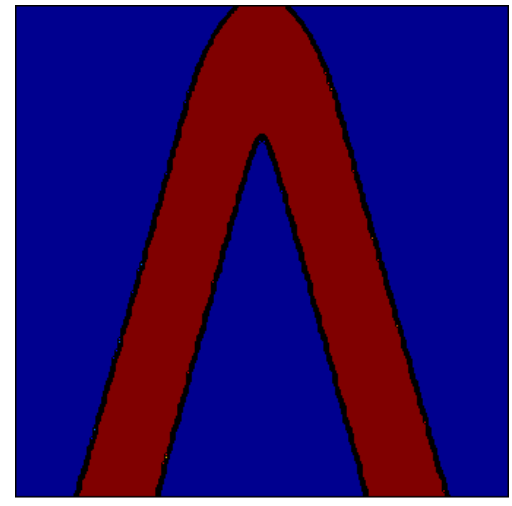

(b) $[\theta]=\left[-100^{\circ},-80^{\circ}\right]$

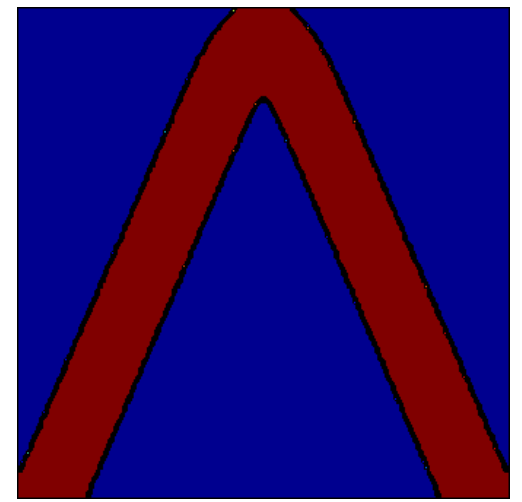

(c) $[\theta]=\left[-110^{\circ},-70^{\circ}\right]$

Figure. 5 The RTO of simple column using interval optimization

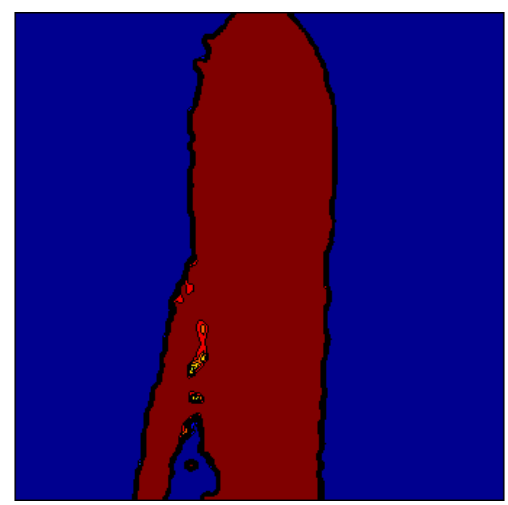

(a) $[\theta]=\left[-95^{\circ},-85^{\circ}\right]$

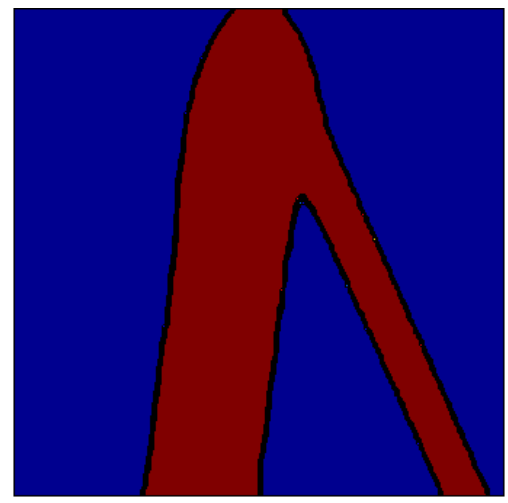

(b) $[\theta]=\left[-100^{\circ},-80^{\circ}\right]$

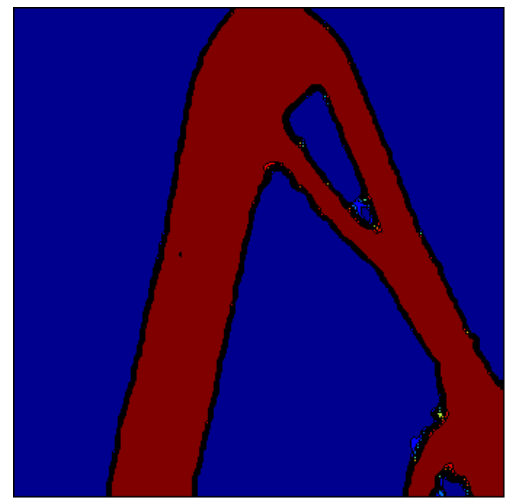

(c) $[\theta]=\left[-110^{\circ},-70^{\circ}\right]$

Figure. 6 The RTO of simple column based on minimax optimization 
In the deterministic case, the force is fixed in the vertical direction, so all the material is distributed along the vertical direction. However, this structure is unstable, because its lateral stiffness is relatively very

small, which may produce larger deformation when there is a perturbation from the force direction. The topology of the interval optimization is much more stable than the deterministic one, because the triangle structure provides larger lateral stiffness. With the increase of the uncertainty level, the angle in the top side of the structure becomes larger, in order to improve the lateral stiffness to bear the loading. When the uncertainty level achieves $20^{\circ}$, the angle in the top will not change, since the two support points in the bottom side have reached the two end points of the design domain. The results of the RTO by using the minimax model based on the vertices are given in Fig. 6. The minimax model is difficult to convergence, so the convergence criterion of the optimization procedure is that the maximum allowable iteration iterations are 500. Therefore, the optimized results of the minimax model will contain some intermediate densities.
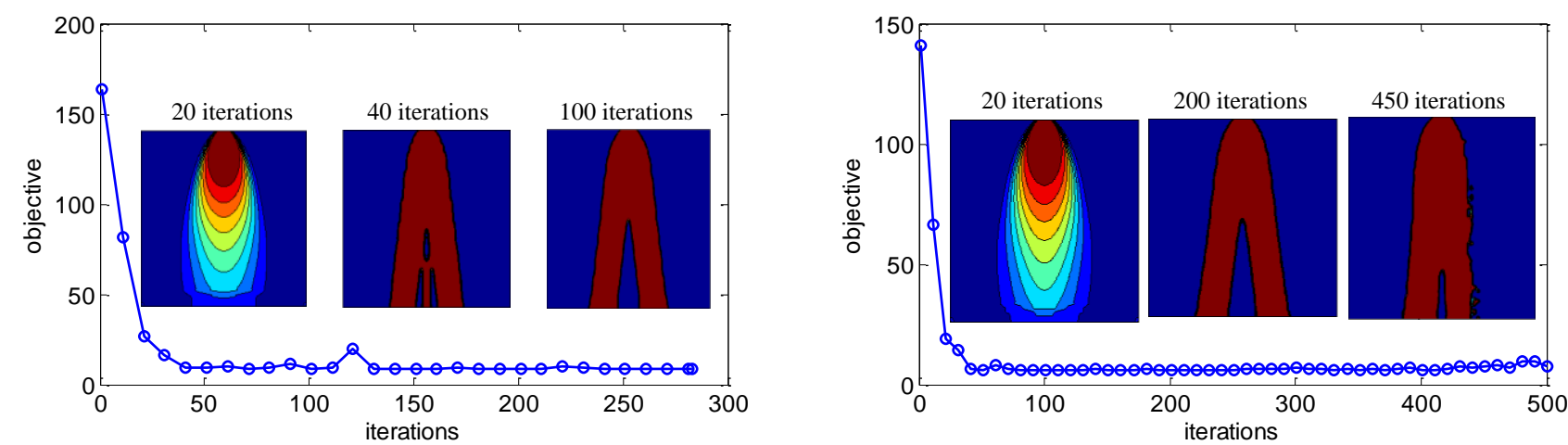

(a) $[\theta]=\left[-95^{\circ},-85^{\circ}\right]$
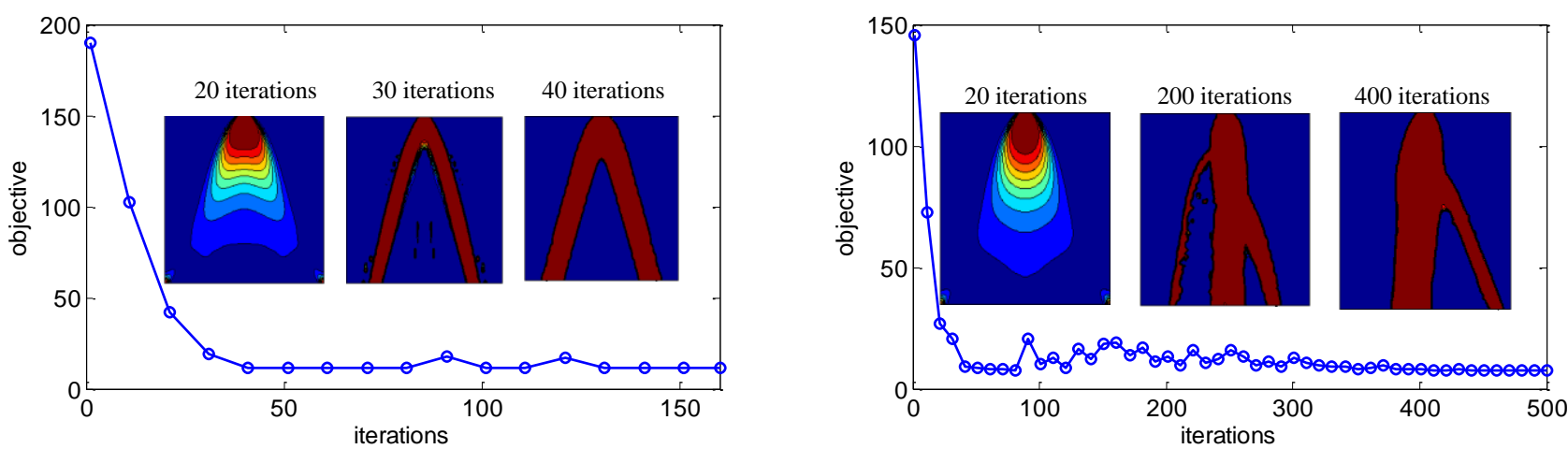

(b) $[\theta]=\left[-100^{\circ},-80^{\circ}\right]$ 

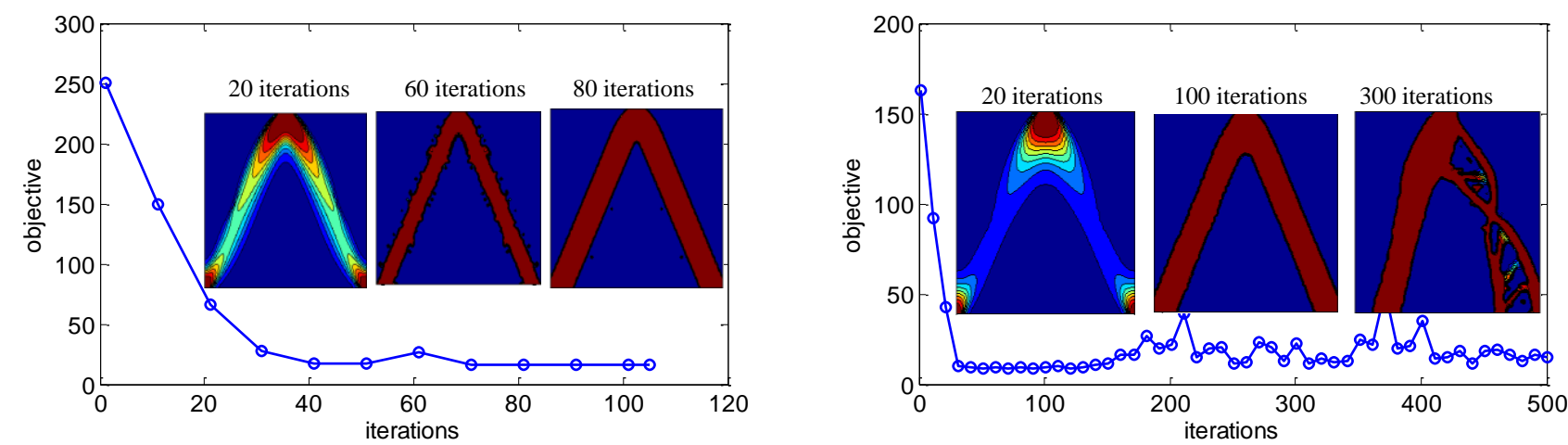

(c) $[\theta]=\left[-110^{\circ},-70^{\circ}\right]$

Figure. 7 Iteration histories of simple column (left: Interval optimization; right: minimax optimization)

The iteration histories of the interval optimization and minimax optimization model are shown in Fig. 7. In general, the interval optimization topologically converges to the optimized design within 100 iterations and the rest iterations will be used to locally adjust the distribution of the material. Figure 7 also shows that the interval optimization can be processed stably without experiencing oscillation until the optimized design is obtained. due to the differentiability of the interval uncertainty optimization of the structure. The iteration history shows that the minimax optimization contains many waves in the optimization procedure and the iteration does not stop until the maximum iteration (500 iterations) is reached. Hence the minimax optimization model may be unstable.

The compliances under the worst case of loading conditions are given in Table 1, which shows that the deterministic solutions of the topology optimization problem produce larger compliance than that of RTO. In Table 1, the solutions for both the deterministic and uncertain optimization problems are obtained by using a scanning method to find the maximum of the objective function within the intervals. It can be seen that with the increasing of the uncertainty level the compliance of the deterministic optimization problem increases obviously, because the loading will have a notable impact on the structural stability at the transverse direction, while the RTO based on interval optimization keeps the compliance to be minimized under the worst case. The interval optimization also provides smaller compliance than the minimax optimization model. The values in the bracket are compliance computed by the interval method. It is noted that the compliance obtained by the interval method is larger the actual compliance obtained by the scanning method. However, since here we only use the compliance computed by the interval method as an intermediary to obtain the optimal solution, it will not affect the final result.

Table 1 The compliance of simple column under the worst case condition

\begin{tabular}{lccc}
\hline & {$[\theta]=\left[-95^{\circ},-85^{\circ}\right]$} & {$[\theta]=\left[-100^{\circ},-80^{\circ}\right]$} & {$[\theta]=\left[-110^{\circ},-70^{\circ}\right]$} \\
\hline Deterministic & 10.62 & 21.93 & 65.46 \\
\hline
\end{tabular}




\begin{tabular}{lccc}
\hline RTO by Interval & $5.98(8.54)$ & $6.88(11.24)$ & $8.86(16.42)$ \\
\hline RTO by Minimax & 7.42 & 7.39 & 12.05 \\
\hline
\end{tabular}

\subsection{Cantilever beam design}

Figure 8 shows the cantilever beam structure where the volume fraction constraint is set to 0.3 . There are two forces applied at the two corners of the right edge, as shown in Fig. 9. In the deterministic condition, the magnitudes of both the two forces are equal to 1 . Considering the interval uncertainties, the magnitude of the two forces is set as: 1) $\mathbf{F}=[0.95,1.05]^{2}$, 2) $\mathbf{F}=[0.9,1.1]^{2}$, and 3) $\mathbf{F}=[0.8,1.2]^{2}$. The mesh size is 60 by 30 , and all other parameters about the RTO are the same as the above simple column design.

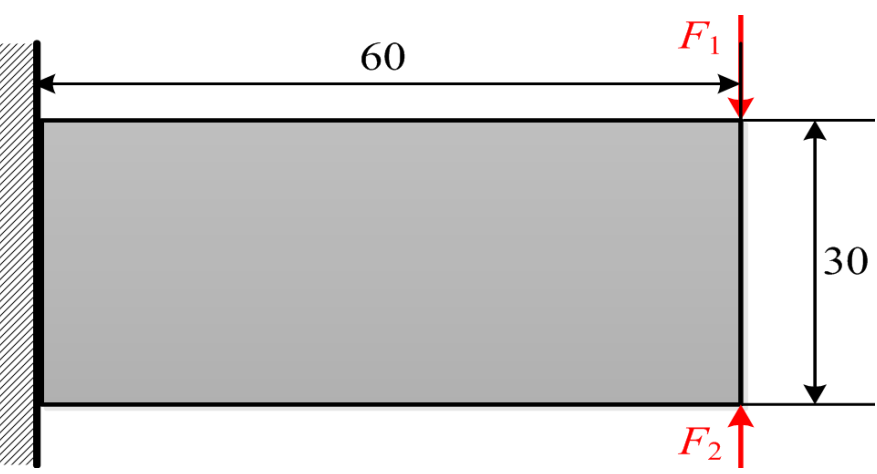

Figure. 8 Design domain

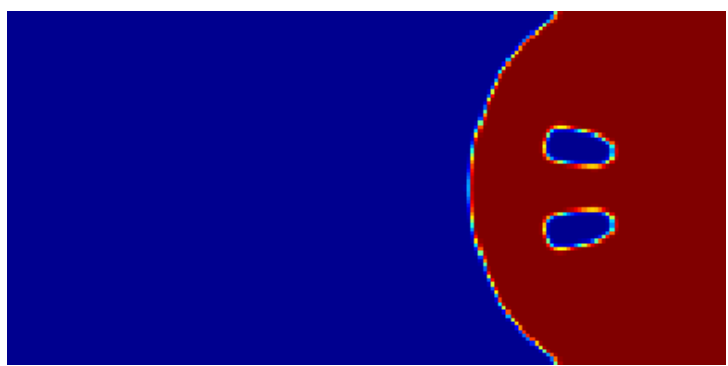

Figure. 9 Deterministic optimization design

The deterministic optimization result is shown in Fig. 9. When the two forces are exactly equivalent in magnitude but applied in the opposite directions, the stress of the beam will not be transmitted to the left side of the domain, so the materials will only be distributed in the right side of the domain. However, such structure is unstable, due to the infinite displacements once the two forces are not exactly equivalent.



(a) $\mathbf{F}=[0.95,1.05]^{2}$

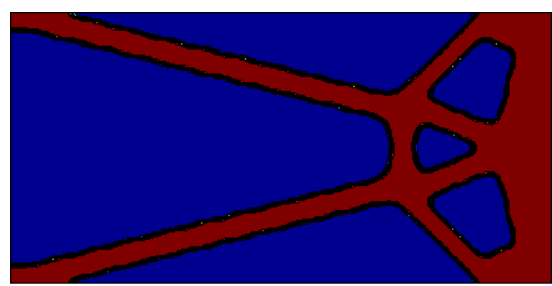

(b) $\mathbf{F}=[0.9,1.1]^{2}$

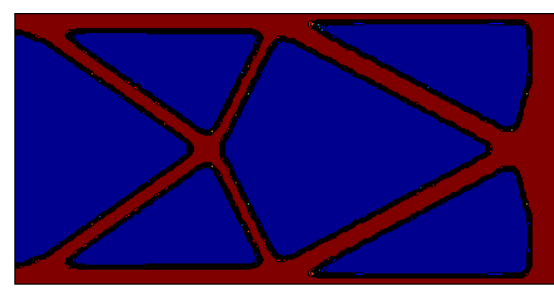

(c) $\mathbf{F}=[0.8,1.2]^{2}$

Figure. 10 The RTO of cantilever beam using interval optimization
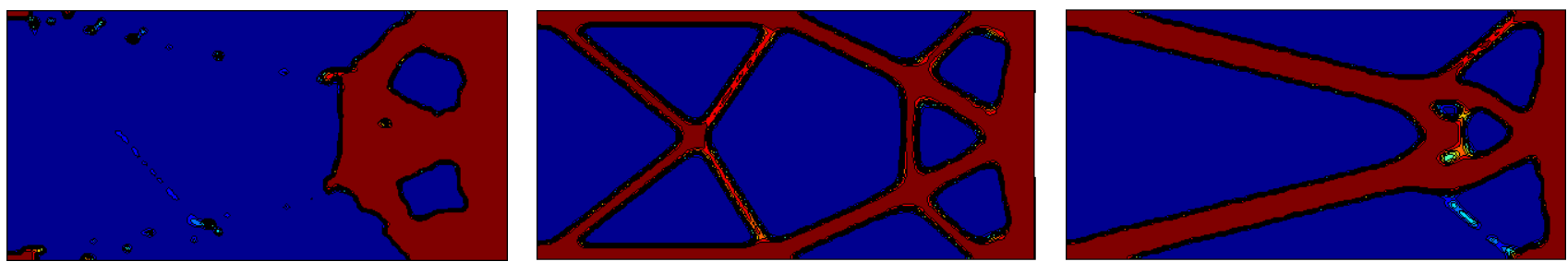

(a) $\mathbf{F}=[0.95,1.05]^{2}$
(b) $\mathbf{F}=[0.9,1.1]^{2}$
(c) $\mathbf{F}=[0.8,1.2]^{2}$

Figure. 11 The RTO of cantilever beam using minimax optimization

The results of the RTO by using interval method under different uncertain conditions are given in Fig. 10. The two types of optimization methods provide quite different solution. It can be found that there are some connections between the left side and the right side when the uncertain level is $5 \%$, which can avoid infinite displacements. When the uncertain level increases to $10 \%$, the connecting bars between the left side and the right domain become thicker. When the uncertain range reaches to $20 \%$, there are more bars connecting the left side and right domain, which means that more material has been transformed from the right to the left of the design domain. There are more loads transferred to the left side when the uncertainty level increases, so more materials will be distributed in the left domain. The results of RTO by using the minimax optimization model are shown in Fig. 11. It can be found that the topologically optimized structure under very small level of uncertainty (e.g. $5 \%$ ) is unstable, similar to the deterministically designed structure (Fig. 8). The other two cases (10\% and $20 \%$ uncertain levels) give more stable topological designs, but there are some intermediate densities in the final results. Since the minimax optimization is unstable (Fig. 12), the optimization procedure is not converged until the 500 iterations have been reached. Therefore, the final results involve more intermediate densities.
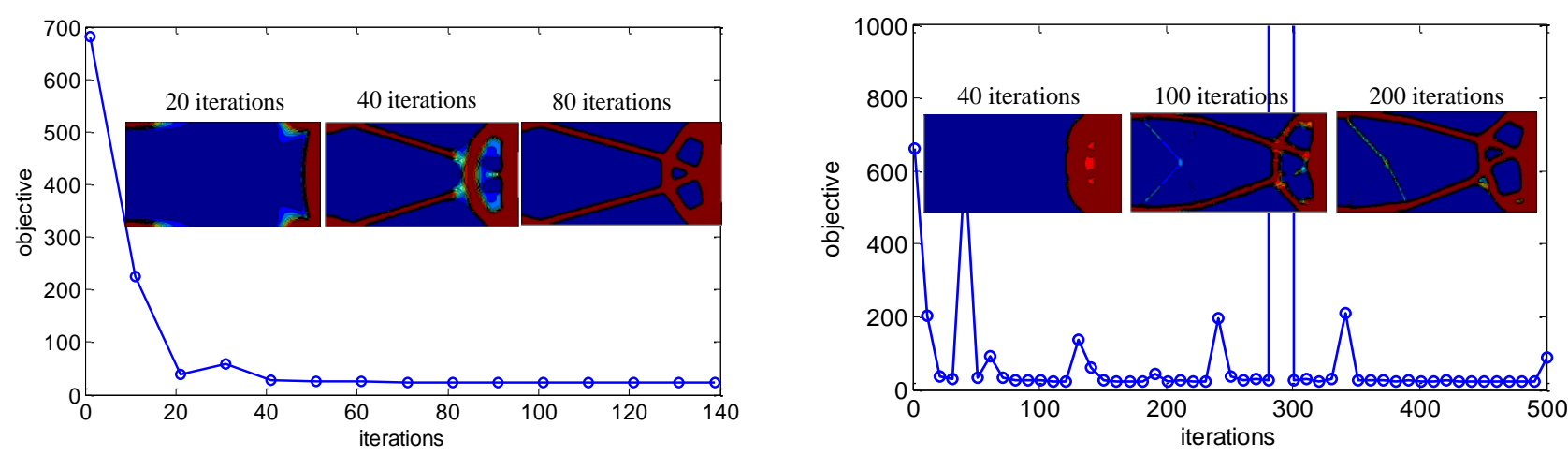

(a) $\mathbf{F}=[0.95,1.05]^{2}$


(b) $\mathbf{F}=[0.9,1.1]^{2}$ 

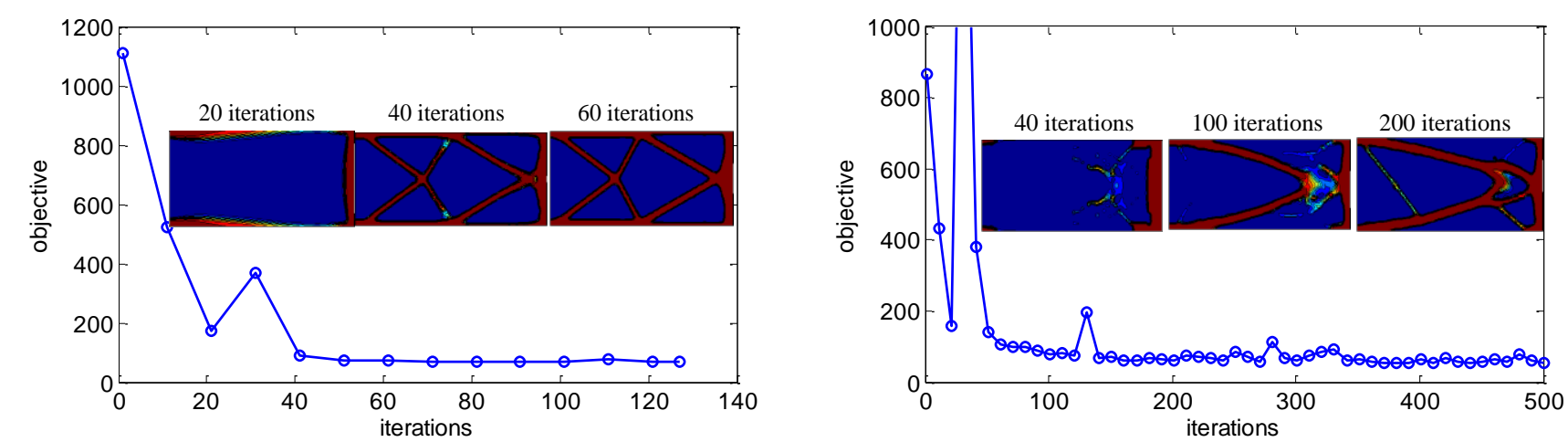

(c) $\mathbf{F}=[0.8,1.2]^{2}$

Figure.12 Iteration histories of cantilever beam (left: interval optimization; right: minimax optimization)

The iteration histories of the cantilever beam are shown in Fig. 11. The interval uncertainty optimization problem of the structure is more stable than the minimax optimization. At the initial 40 iterations of the interval optimization, there are oscillations, which are caused by the drastic change of structural topology at the initial stage of evolution. The interval optimization converges after 40 iterations (except the $10 \%$ uncertain level which converges after 200 iterations), so the computation is more efficient for the proposed interval optimization method. Besides the unstablility, the iteration histories of minimax optimization involves obvious fluctuations and difficult to converge until the maximum iterations is reached.

Table 2 The compliance of cantilever beam under the worst-case condition

\begin{tabular}{lccc}
\hline & $\mathbf{F}=[0.95,1.05]^{2}$ & $\mathbf{F}=[0.9,1.1]^{2}$ & $\mathbf{F}=[0.8,1.2]^{2}$ \\
\hline Deterministic & $3.67 \mathrm{e} 11$ & $1.43 \mathrm{e} 12$ & $5.46 \mathrm{e} 12$ \\
\hline RTO by Interval & $21.24(23.70)$ & $28.14(33.36)$ & $49.92(69.31)$ \\
\hline RTO by Minimax & 120.06 & 30.76 & 57.26 \\
\hline
\end{tabular}

The detailed objective values of the design are given in Table 2. The compliances of the worst case of the deterministic optimization tend to be infinite, because there is no connection between the left and the right sides of the domain. The fixed points are located in the left side but the external forces are located in the right side. The compliance of RTO is much smaller than the deterministic topology optimization. At the same time, the interval optimization gives smaller compliance values than the minimax optimization.

\subsection{Michell-type structure design}

The boundary conditions and loads for the Michell-type structure are given in Fig. 13. The volume fraction is limited to 0.3 , and the magnitude of the three forces are $F_{1}=1, F_{2}=2$, and $F_{3}=1$. The force direction is assumed to be $\theta_{1}=\theta_{2}=\theta_{3}=-90^{\circ}$ in the deterministic design. For the uncertain conditions, the 
force direction is assumed to be: 1) $\boldsymbol{\theta}=\left[-95^{\circ},-85^{\circ}\right]^{3}$; 2) $\boldsymbol{\theta}=\left[-100^{\circ},-80^{\circ}\right]^{3}$; 3) $\boldsymbol{\theta}=\left[-110^{\circ},-70^{\circ}\right]^{3}$. The mesh size is set as 120 by 50, and all other parameters about the RTO are the same as the simple column design.

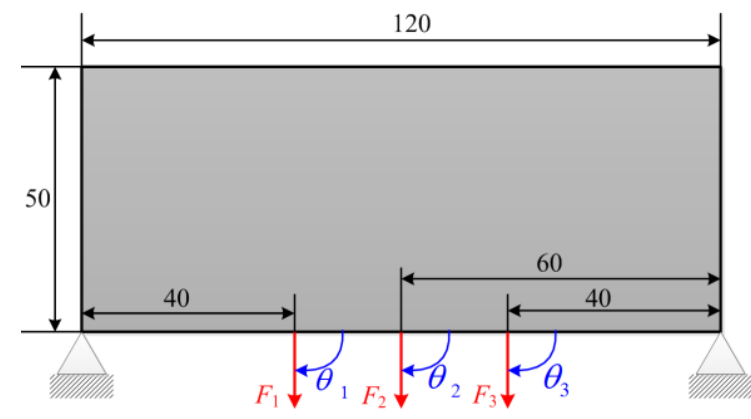

Figure. 13 Design domain

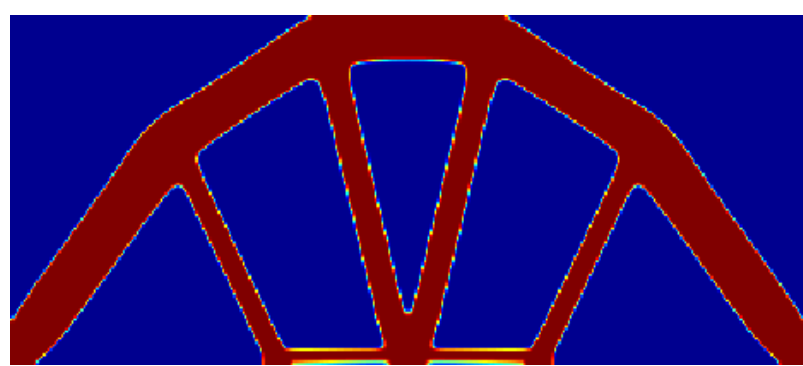

Figure. 14 Deterministic design

Figures 14 to 16 show the results of the deterministic topology optimization and RTO by using the interval method and minimax model, respectively. For the deterministic topology optimization, there are no materials between the two end points and the locations of $F_{1}$ and $F_{3}$. Figure 15 shows that two crossing structures are occurred in the left bottom and right bottom of the domain when the uncertainty is 5 , which may be useful to bear lateral force induced by the perturbation of the force direction. When the uncertainty approaches to $10^{\circ}$, it can be found that the two crossing structures move downward. When the uncertainty gets $20^{\circ}$, the crossing structures have moved to the bottom of the design domain, and they have degenerated to the direct linked bar.

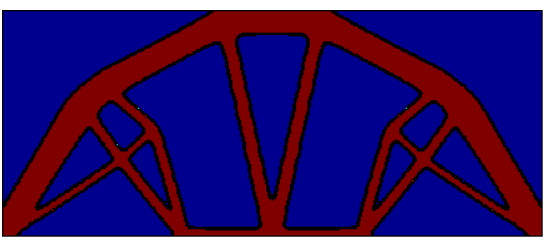

(a) $\boldsymbol{\theta}=\left[-95^{\circ},-85^{\circ}\right]^{3}$

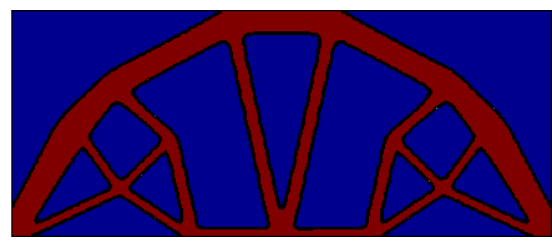

(b) $\boldsymbol{\theta}=\left[-100^{\circ},-80^{\circ}\right]^{3}$

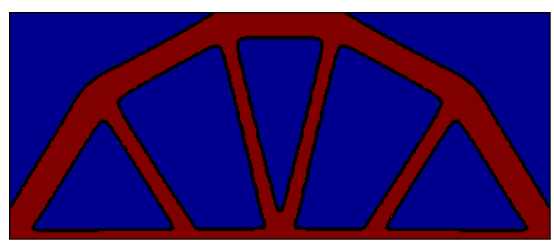

(c) $\boldsymbol{\theta}=\left[-110^{\circ},-70^{\circ}\right]^{3}$

Figure. 15 RTO of Mitchell-type structure using interval optimization

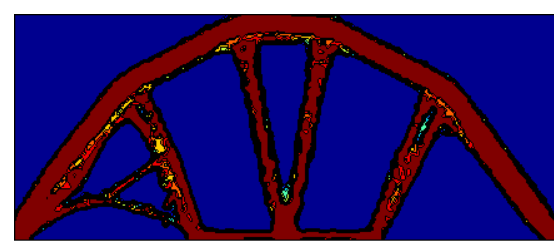

(a) $\boldsymbol{\theta}=\left[-95^{\circ},-85^{\circ}\right]^{3}$

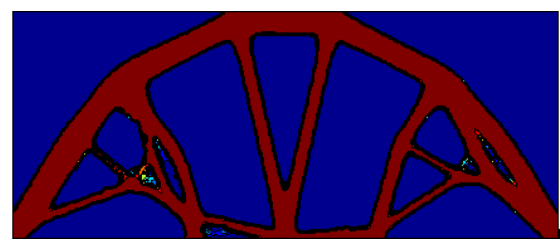

(b) $\boldsymbol{\theta}=\left[-100^{\circ},-80^{\circ}\right]^{3}$

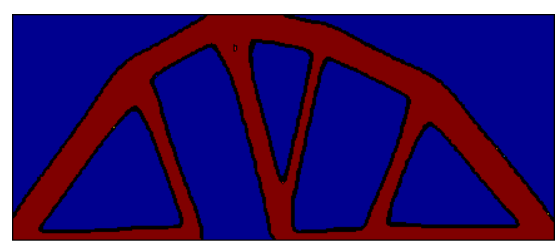

(c) $\boldsymbol{\theta}=\left[-110^{\circ},-70^{\circ}\right]^{3}$

Figure. 16 RTO of Mitchell-type structure using minimax optimization

Figure 16 gives unsymmetrical topological design for the minimax optimization and many intermediate densities occur for the cases under $5^{\circ}$ and $10^{\circ}$ uncertainties. Therefore, the minimax optimization may not be convergent, which can be further indicated by the iteration history (Fig. 17). 

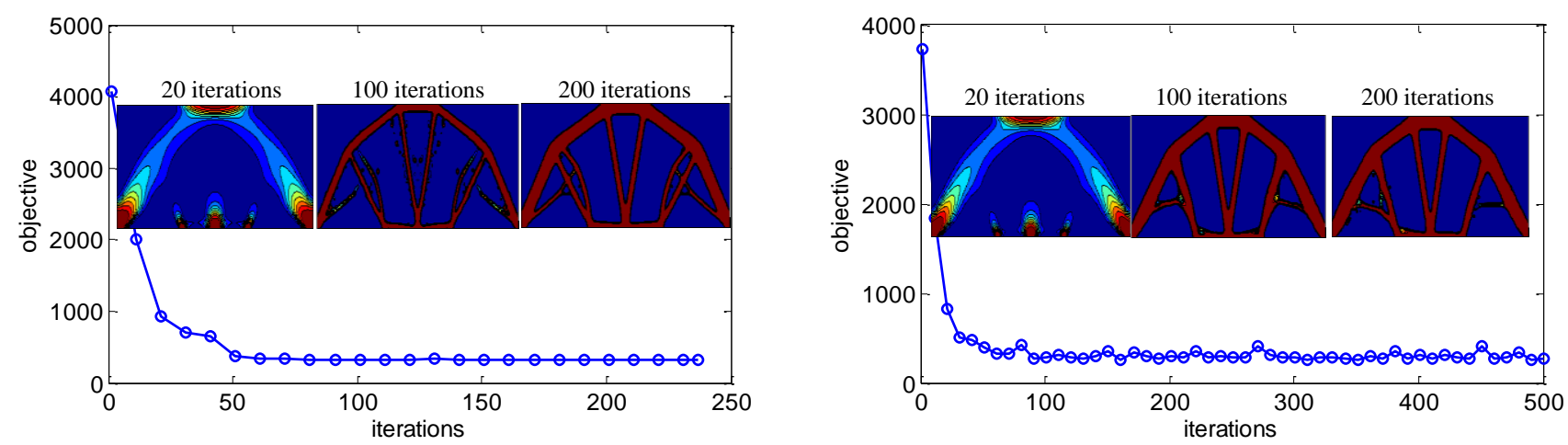

(a) $\boldsymbol{\theta}=\left[-95^{\circ},-85^{\circ}\right]^{3}$
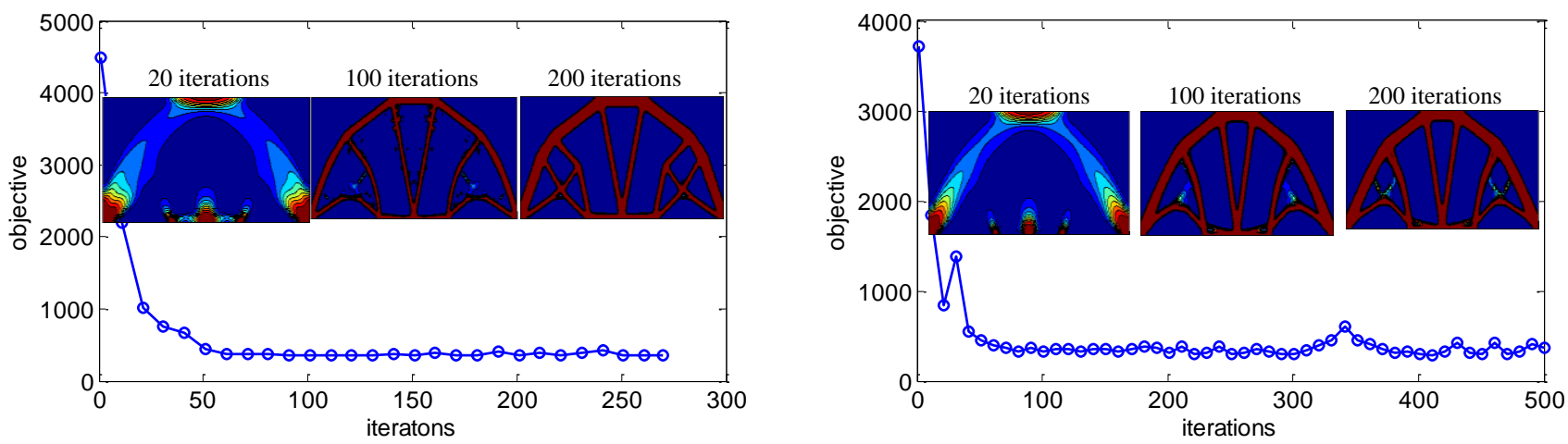

(b) $\boldsymbol{\theta}=\left[-100^{\circ},-80^{\circ}\right]^{3}$
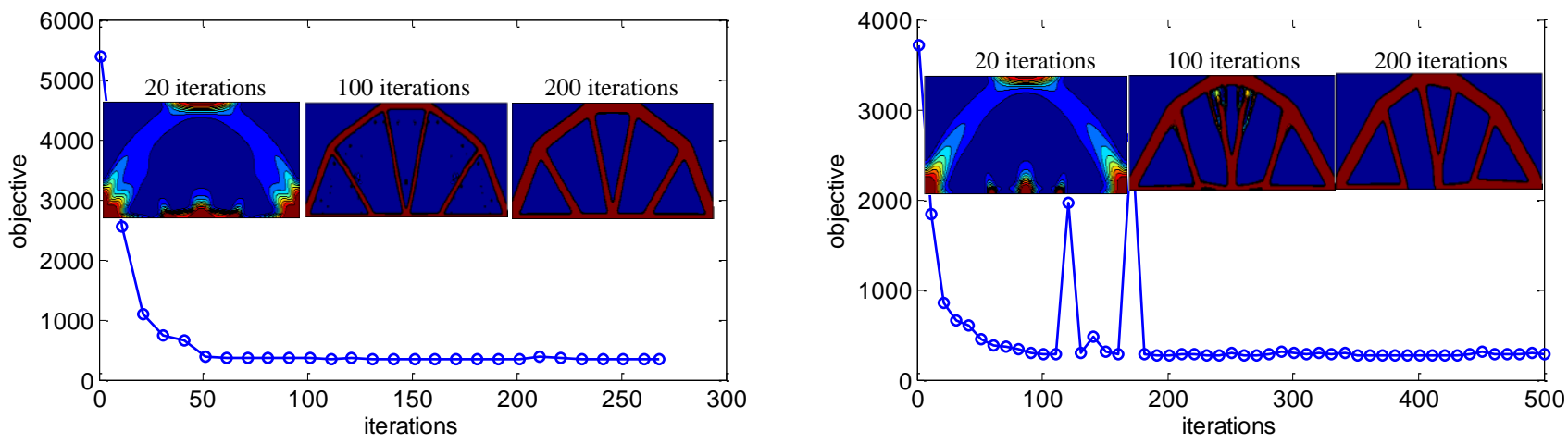

(c) $\boldsymbol{\theta}=\left[-110^{\circ},-70^{\circ}\right]^{3}$

Figure. 17 Iteration histories of Mitchell beam (left: interval optimization; right: minimax optimization)

Figure 17 shows the iteration history of the RTO under different uncertainty levels. After 50 iterations, the variation of the interval objective function is relatively small, and the iteration is stable. However, there are large variations for the objective function of the minimax optimization in the process of the whole optimization, and the optimization does not converge until the maximum iterations are reached.

The detailed objective function values are given in Table 3. The compliance shows that under worst case condition the proposed interval optimization can produce smaller compliance than both the deterministic topology optimization and the minimax optimization.

Table 3 The compliance of Mitchell-type under the worst condition 


\begin{tabular}{lccc}
\hline & $\boldsymbol{\theta}=\left[-95^{\circ},-85^{\circ}\right]^{3}$ & $\boldsymbol{\theta}=\left[-100^{\circ},-80^{\circ}\right]^{3}$ & $\boldsymbol{\theta}=\left[-110^{\circ},-70^{\circ}\right]^{3}$ \\
\hline Deterministic & 310 & 556 & 1503 \\
\hline RTO by Interval & $255(320)$ & $267(354)$ & $250(350)$ \\
\hline RTO by & 424 & 321 & 278 \\
Minimax & & & \\
\hline
\end{tabular}

To consider both the uncertain magnitude and the direction of loads simultaneously another case of Mitchell-type structure is used. The loads are $F_{2}=[1.8,2.2]$ and $F_{1}=F_{3}=1$ with direction $\theta_{1}=\left[-100^{\circ},-80^{\circ}\right]$. $\theta_{2}=-90^{\circ}$ and $\theta_{3}=\left[-100^{\circ},-80^{\circ}\right]$. Using the interval optimization method and the minimax optimization method to solve this problem the optimized topologies are shown in Fig. 18. The iteration history is shown in Fig. 19, which shows that the proposed interval topology optimization method is more smooth and stable than the minimax optimization. The compliance of the interval topology optimization is 287 that is smaller than the compliance of minimax optimization 321 . Hence the proposed interval optimization is better.

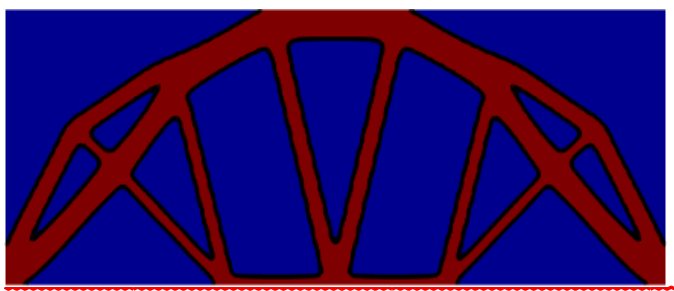

(a) Interval optimization

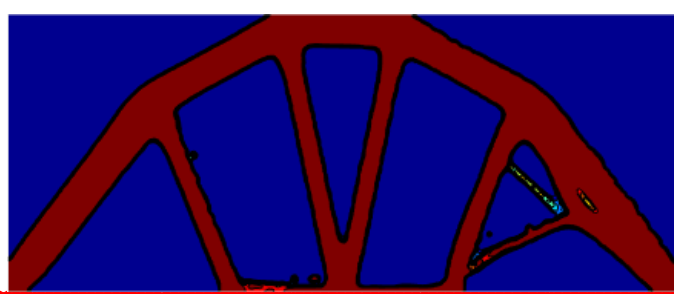

(b) Minimax optimization

Figure 18. RTO of Mitchell beam under both uncertain magnitude and direction of loads

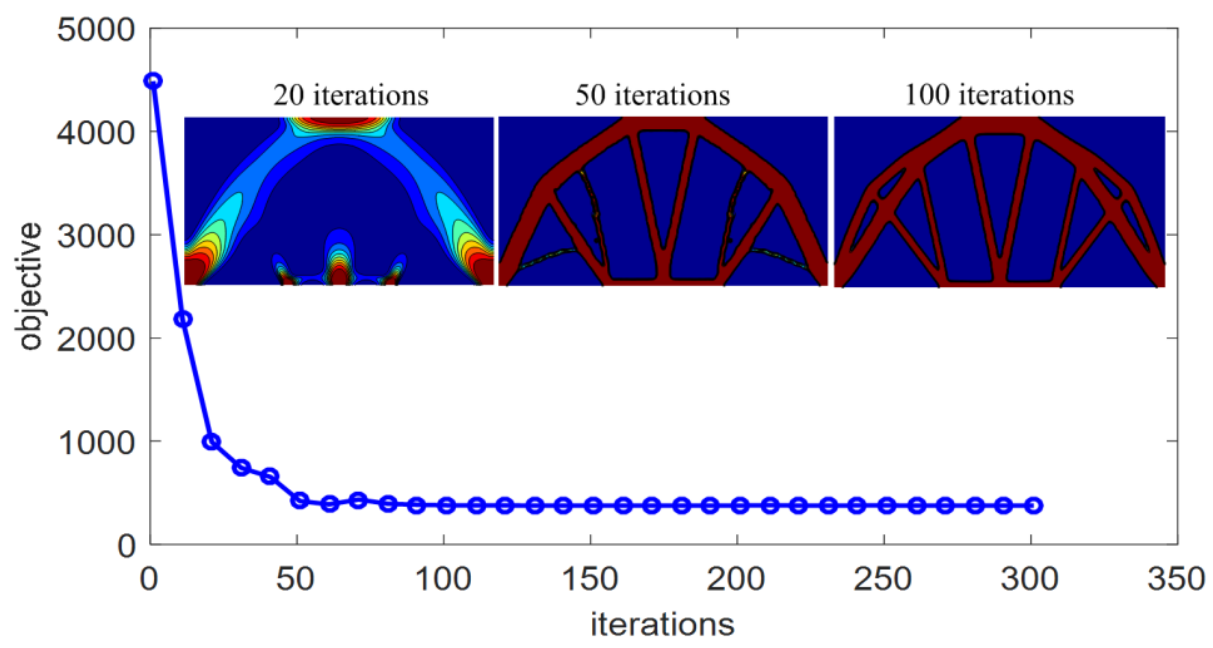

(a) Interval optimization 


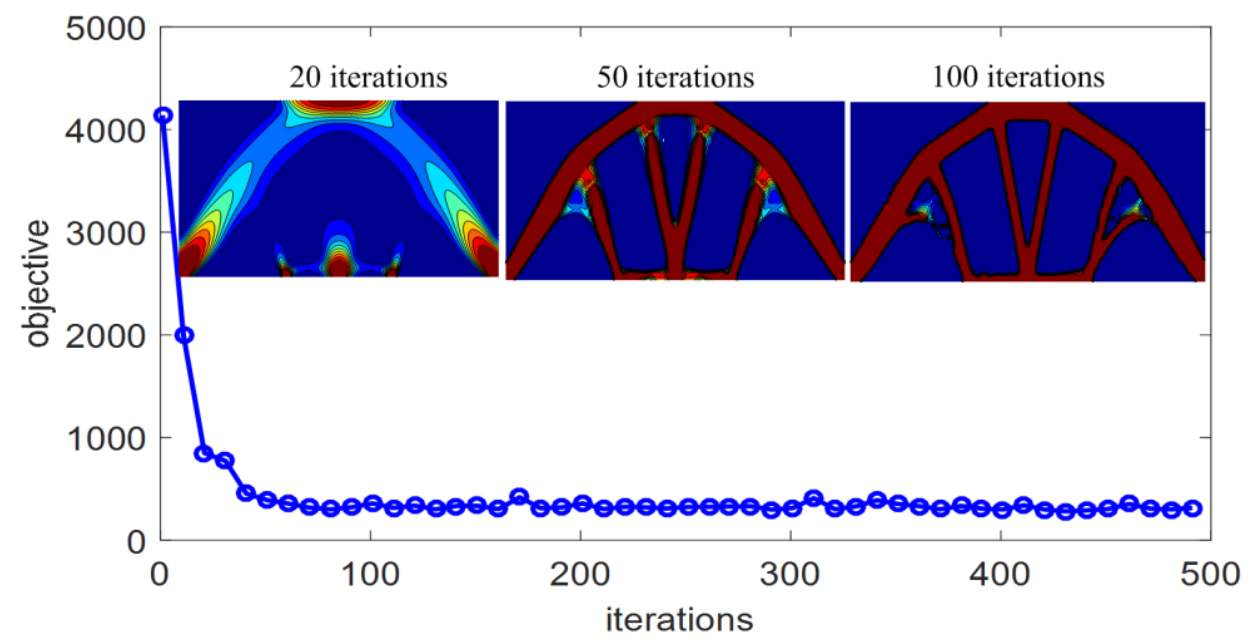

(b) Minimax optimization

Figure. 19 Iteration histories of Mitchell beam under both uncertain magnitude and direction of loads

\section{Conclusions}

This paper has proposed a new robust topology optimization method for structures with uncertain-butbounded parameters. To avoid the nested double-loop optimization procedure which is computationally expansive in the interval optimization, the Chebyshev interval method has been applied to the RTO design problems. Since the Chebyshev interval method is non-intrusive, it is preferable for handling the complex models and even black-box models. To take advantage of the gradient-based optimization algorithms, a numerical scheme is developed to evaluate the sensitivity of the interval objective function with respect to the design variables. The interval arithmetic is expressed by using the sign function and then the first-order derivatives of the interval function can be obtained. Several numerical examples have been used to show the effectiveness of the proposed interval RTO method, compared with the minimax optimization model based on the vertices of uncertain parameters. From these examples, it can be found that it is necessary to consider the uncertain parameters in the topology optimization of continuum structures, because even a relative small variation of loads may lead to obvious change of the structural performance.

\section{Reference}

[1] Bendsoe MP, Sigmund O. Topology Optimization: Theory, Methods, and Applications Berlin, Heidelberg: Springer; 2003.

[2] Bendsøe MP, Kikuchi N. Generating optimal topology in structural design using a homogenization method. Computer Methods in Applied Mechanics and Engineering. 1988;71:197-224.

[3] Zhou M, Rozvany GIZ. The COC algorithm, Part II: topological, geometry and generalized shape optimization. Computer Methods in Applied Mechanics and Engineering. 1991;89:197-224.

[4] Bendsoe MP, Sigmund O. Material interpolation schemes in topology optimization. Archive of Applied Mechanics. 1999;69:635-54. 
[5] Sethian JA, Wiegmann A. Structural boundary design via level set and immersed interface methods. Journal of Computational Physics. 2000;163:227-46.

[6] Wang MY, Wang XM, Guo DM. A level set method for structural topology optimization. Computer Methods in Applied Mechanics and Engineering. 2003;192:227-46.

[7] Allaire G, Jouve F. Structural optimization using sensitivity analysis and a level-set method. Journal of Computational Physics. 2004;194:363-93.

[8] Xie YM, Steven GP. A simple evolutionary procedure for structural optimization. Computer \& Structures. 1993;49:885-96.

[9] Huang XD, Xie YM. A further review of ESO type methods for topology optimization. Structural and Multidisciplinary Optimization. 2010;41:671-83.

[10] Asadpoure A, Tootkaboni M, Guest JK. Robust topology optimization of structures with uncertainties in stiffness - Application to truss structures. Computer \& Structures. 2011;89:1131-41.

[11] Valdebenito MA, Schuëller GI. A survey on approaches for reliability-based optimization. Structural and Multidisciplinary Optimization. 2010;42:645-63.

[12] Schuëller GI, Jensen HA. Computational methods in optimization considering uncertainties - an overview. Computer Methods in Applied Mechanics and Engineering 2008;198:2-13.

[13] Du X, Sudjianto A, Huang B. Reliability-Based Design With the Mixture of Random and Interval Variables. Journal of Mechanical Design. 2005;127:1068.

[14] Jiang C, Long XY, Han X, Tao YR, Liu J. Probability-interval hybrid reliability analysis for cracked structures existing epistemic uncertainty. Engineering Fracture Mechanics. 2013;112-113:148-64.

[15] Gao W, Song C, Tin-Loi F. Static response and reliability analysis of structural systems with random and interval properties. IOP Conference Series: Materials Science and Engineering. 2010;10:012200.

[16] Beyer H-G, Sendhoff B. Robust optimization - A comprehensive survey. Computer Methods in Applied Mechanics and Engineering. 2007;196:3190-218.

[17] Li M, Azarm S. Multiobjective collaborative robust optimization with interval uncertainty and interdisciplinary uncertainty propagation. Journal of Mechanical Design. 2008;130:081402.

[18] Apley DW, Liu J, Chen W. Understanding the effects of model uncertainty in robust design with computer experiments. Journal of Mechanical Design. 2006;128:945.

[19] Zaman K, McDonald M, Mahadevan S, Green L. Robustness-based design optimization under data uncertainty. Structural and Multidisciplinary Optimization. 2011;44:183-97.

[20] Kharmanda G, Olhoff N, Mohamed A, Lemaire M. Reliability-based topology optimization. structural and Multidisciplinary Optimization. 2004;26:295-307.

[21] Patel NM, Renaud JE, Agarwal H, Tovar A. Reliability based topology optimization using the hybrid cellular automaton method. 46th AIAA/ASME/ASCE/AHS/ASC structures, structural dynamics and materials conference Austin, Texas2005.

[22] Luo Y, Kang Z, Luo Z, Li A. Continuum topology optimization with non-probabilistic reliability constraints based on multi-ellipsoid convex model. Structural and Multidisciplinary Optimization. 2008;39:297-310.

[23] Ben-Haim Y, Elishakoff I. Convex models of uncertainty in applied mechanics, Amsterdam: Elsevier; 1990. 
[24] Elishakoff I. Three versions of the finite element method based on concepts of either stochasticity, fuzziness or anti-optimization. Applied Mechanics Review. 1998;51:209-18.

[25] Kang Z, Luo Y. Non-probabilistic reliability-based topology optimization of geometrically nonlinear structures using convex models. Computer Methods in Applied Mechanics and Engineering. 2009;198:3228-38.

[26] Maute K. Topology optimization under uncertainty. Topology optimization in structural and continuum mechanics, Vienna: Springer; 2014. p. 457-71.

[27] Sigmund O. Manufacturing tolerant topology optimization. Acta Mechanica Solida Sinica. 2009;25.

[28] Wang F, Lazarov BS, Sigmund O. On projection methods, convergence and robust formulations in topology optimization. Structural and Multidisciplinary Optimization. 2010;43:767-84.

[29] Guest JK, Igusa T. Structural optimization under uncertain loads and nodal locations. Computer Methods in Applied Mechanics and Engineering. 2008;198:116-24.

[30] Lazarov BS, Schevenels M, Sigmund O. Topology optimization with geometric uncertainties by perturbation techniques. International Journal for Numerical Methods in Engineering. 2012;90:1321-36.

[31] Tootkaboni M, Asadpoure A, Guest JK. Topology optimization of continuum structures under uncertainty - A Polynomial Chaos approach. Computer Methods in Applied Mechanics and Engineering. 2012;201-204:263-75.

[32] Zhao J, Wang C. Robust structural topology optimization under random field loading uncertainty. Structural and Multidisciplinary Optimization. 2014;50:517-22.

[33] Chen S, Chen W, Lee S. Level set based robust shape and topology optimization under random field uncertainties. Structural and Multidisciplinary Optimization. 2010;41:507-24.

[34] Jansen M, Lombaert G, Diehl M, Lazarov BS, Sigmund O, Schevenels M. Robust topology optimization accounting for misplacement of material. Structural and Multidisciplinary Optimization. 2013;47:317-33.

[35] Zhao J, Wang C. Robust topology optimization under loading uncertainty based on linear elastic theory and orthogonal diagonalization of symmetric matrices. Computer Methods in Applied Mechanics and Engineering. 2014;273:204-18.

[36] Wang NF, Yang YW. Structural design optimization subjected to uncertainty using fat Bezier curve. Computer Methods in Applied Mechanics and Engineering. 2009;199:210-9.

[37] Takezawa A, Nii S, Kitamura M, Kogiso N. Topology optimization for worst load conditions based on the eigenvalue analysis of an aggregated linear system. Computer Methods in Applied Mechanics and Engineering. 2011;200:2268-81.

[38] Csébfalvi A. A New Theoretical approach for robust truss optimization with uncertain load directions. Mechanics Based Design of Structures and Machines. 2014;42:442-53.

[39] Csébfalvi A. Angel method for discrete optimization problems. Periodica Polytechnica Civil Engineering. 2007; 51:37.

[40] Jiang C, Han X, Liu GR. Optimization of structures with uncertain constraints based on convex model and satisfaction degree of interval. Computer Methods in Applied Mechanics and Engineering. 2007;196:4791-800.

[41] Jiang C, Han X, Liu GP. Uncertain optimization of composite laminated plates using a nonlinear interval number programming method. Computers \& Structures. 2008;86:1696-703.

[42] Liu N, Gao W, Song C, Zhang N, Pi Y-L. Interval dynamic response analysis of vehicle-bridge interaction system with uncertainty. Journal of Sound and Vibration. 2013;332:3218-31. 
[43] Gao W, Wu D, Song C, Tin-Loi F, Li X. Hybrid probabilistic interval analysis of bar structures with uncertainty using a mixed perturbation Monte-Carlo method. Finite Elements in Analysis and Design. 2011;47:643-52.

[44] Gao W, Song C, Tin-Loi F. Probabilistic interval analysis for structures with uncertainty. Structural Safety. 2010;32:191-9.

[45] Moore RE. Interval analysis Englewood Cliffs, New Jersey: Prentice-Hall; 1966.

[46] Wu J, Zhang Y, Chen L, Luo Z. A Chebyshev interval method for nonlinear dynamic systems under uncertainty. Applied Mathematical Modelling. 2013;37:4578-91.

[47] Wu J, Luo Z, Zhang Y, Zhang N, Chen L. Interval uncertain method for multibody mechanical systems using Chebyshev inclusion functions. International Journal for Numerical Methods in Engineering. 2013;95:608-30.

[48] Barrio R. Performance of the Taylor series method for ODEs/DAEs. Applied Mathematics and Computation. 2005; 163:525-45.

[49] Nedialkov NS, Pryce JD. Solving Differential-Algebraic Equations by Taylor Series (I) Computing Taylor Coefficients. BIT. 2005:001-30.

[50] Berz M, Makino K. Rigorous Global Search using Taylor Models. Proceedings of the 2009 conference on Symbolic numeric computation Kyoto, Japan2009. p. 11-20.

[51] Makino K, Berz M. Suppression of the wrapping effect by Taylor model-based validated integrators. Michigan State University; 2004.

[52] Wu J, Luo Z, Zhang Y, Zhang N. An interval uncertain optimization method for vehicle suspensions using Chebyshev metamodels. Applied Mathematical Modelling. 2014;38:3706-23.

[53] Wu J, Luo Z, Zhang N, Zhang Y. A new interval uncertain optimization method for structures using Chebyshev surrogate models. Computers \& Structures. 2015;146:185-96.

[54] Sigmund O. A 99 line topology optimization code written in matlab. Structural and Multidisciplinary Optimization. 2001;21:120-7.

[55] Bendsoe MP. Optimization of structureal topology, shape and material. Berlin, Heidelberg: New York: Springer; 1995.

[56] Svanberg K. The method of moving asymptotes - a new method for structural optimization. International Journal for Numerical Methods in Engineering. 1987;24:359-73.

[57] Bourdin B. Filters in topology optimization. International Journal for Numerical Methods in Engineering. 2001;50:2143 - 58 .

[58] Jaulin L. Applied Interval Analysis: with Examples in Parameter and State Estimation, Robust Control and Robotics New York: Springer; 2001.

[59] Svanberg K. Some modelling aspects for the Matlab implementation of MMA. KTH Royal Institute of Technology, Stockholm. 2004. 